\title{
Refill adherence and self-reported adverse drug reactions and sub-therapeutic effects: a population-based study
}

\author{
Khedidja Hedna, Staffan Hägg, Karolina Andersson Sundell, Max Petzold and Katja M \\ Hakkarainen
}

\section{Linköping University Post Print}

\section{Tweet}

N.B.: When citing this work, cite the original article.

Original Publication:

Khedidja Hedna, Staffan Hägg, Karolina Andersson Sundell, Max Petzold and Katja M Hakkarainen, Refill adherence and self-reported adverse drug reactions and sub-therapeutic effects: a population-based study, 2013, Pharmacoepidemiology and Drug Safety, (22), 12, 1317-1325.

http://dx.doi.org/10.1002/pds.3528

Copyright: Wiley

http://eu.wiley.com/WileyCDA/

Postprint available at: Linköping University Electronic Press

http://urn.kb.se/resolve?urn=urn:nbn:se:liu:diva-99892 


\section{Title of the paper}

Refill adherence and self-reported adverse drug reactions and sub-therapeutic effects: A population-based study

\section{Running title}

Refill adherence and reported medication adverse outcomes

\section{Authors}

Khedidja Hedna ${ }^{1,2}$, Staffan Hägg ${ }^{1}$, Karolina Andersson Sundell ${ }^{3}$, Max Petzold ${ }^{4}$, Katja M. Hakkarainen $^{2}$

\section{Institutions}

${ }^{1}$ Division of Drug Research, Department of Medical and Health Sciences, Faculty of Health Sciences, Linköping University, County Council of Östergötland, Sweden

${ }^{2}$ Nordic School of Public Health NHV, Gothenburg, Sweden

${ }^{3}$ Unit of Social Medicine, University of Gothenburg, Gothenburg, Sweden

${ }^{4}$ Centre for Applied Biostatistics, University of Gothenburg, Gothenburg, Sweden

\section{Corresponding author}

Khedidja Hedna, Division of Drug Research, Department of Medical and Health Sciences, Faculty of Health Sciences, Linköping University, County Council of Östergötland, Sweden E-mail: khedidja.hedna@liu.se

Telephone number: +46702027514

\section{Keywords}

Refill adherence; Oversupply; Undersupply; Adverse drug reaction; Therapeutic failure; Selfreport.

\section{Key points}

- $\quad$ Adequate refill adherence was found in two thirds of the medication therapies of antihypertensive, lipid lowering and oral anti-diabetic medications.

- Adverse drug reactions and sub-therapeutic effects were unexpectedly equally commonly reported for medications with adequate refill adherence, oversupply and undersupply.

- A better understanding of patients' refill behaviors and perceived adverse outcomes from their long-term medications is needed and should be considered in improving medication management.

\section{Conflict of interest}

The authors declare that they have no conflict of interest regarding this work.

\section{Financial Disclosure}

This study was conducted as part of the project Drug-Related Morbidity in Sweden (DRUMS). The project was funded through an unrestricted grant from The National Corporation of Swedish Pharmacies (Apoteket AB).

\section{Word count}

2933 


\section{Statement about prior postings and presentations}

The contents of this manuscript are original work and have not been published in scientific journals or presented to public, in whole or in part, prior to or simultaneous with submission of the manuscript to Pharmacoepidemiology and Drug Safety journal. The manuscript has not been through peer-review in other journals. 


\section{ABSTRACT}

Purpose To assess refill adherence to dispensed oral long-term medications among the adult population and to investigate whether the percentages of self-reported adverse drug reactions (ADRs) and sub-therapeutic effects (STEs) differed for medications with adequate refill adherence, oversupply and undersupply.

Method Survey responses on self-reported ADRs and STEs were linked to the Swedish Prescribed Drug Register in a cross-sectional population-based study. Refill adherence to antihypertensive, lipid-lowering and oral anti-diabetic medications was measured using the continuous measure of medication acquisition (CMA). The percentages of self-reported ADRs and STEs were compared between medications with adequate refill adherence (CMA 0.8-1.2), oversupply $(\mathrm{CMA}>1.2)$ and undersupply $(\mathrm{CMA}<0.8)$.

Results The study included 1827 persons and the refill adherence was measured for 3014 antihypertensive, 839 lipid lowering and 253 oral anti-diabetic medications. Overall, $65.7 \%$ of the medications had adequate refill adherence, $21.9 \%$ oversupply, and $12.4 \%$ undersupply. The percentages of self-reported ADRs and STEs were respectively $2.6 \%, 2.7 \%$ and $2.1 \%$ ( $p$ $>0.5)$ for ADRs, and $1.1 \%, 1.6 \%$ and $1.5 \%(p>0.5)$ for STEs.

Conclusion Adequate refill adherence was found in two thirds of the medication therapies. ADRs and STEs were unexpectedly equally commonly reported for medications with adequate refill adherence, oversupply and undersupply. These results suggest that a better understanding of patients' refill behaviors and their perceived medication adverse outcomes is needed and should be considered in improving medication management. The impact of individual and healthcare factors that may influence the association between refill adherence and reported medication adverse outcomes should be investigated in future studies. 


\section{INTRODUCTION}

Suboptimal medication refill adherence among individuals with chronic conditions is a significant clinical problem and has been described as a risk factor for adverse health outcomes and for preventable medication-related morbidities and mortalities. ${ }^{1}$ Adherence to prescribed medications is usually defined as the extent to which an individual acts in accordance with the prescribed dosing interval and dose of prescribed medications. ${ }^{2}$

Oversupply of prescribed medications leads to excessive amounts of medications available to patients and may cause their overuse with a potential risk of adverse drug reactions (ADRs). Undersupply of prescribed medications may lead to their underuse and sub-therapeutic effects (STEs) of these medications.

Earlier studies on adherence conducted in clinical trials have focused on patients who were willing to participate in research studies and received a high attention from health providers. However, the evidence on actual reported medication-related adverse outcomes due to refill non adherence to long-term medications in clinical practice is limited. ${ }^{3}$ In numerous previous population-based studies, refill adherence has been assessed in prescription databases without investigating its association with the ADRs and STEs perceived by patients. ${ }^{4-6}$

To the best of our knowledge, no previous population study assessed the association between refill non adherence to long-term medications and self-reported ADRs and STEs from these medications in a representative sample of the general population. Such evidence is needed for improving the management and outcomes of medication therapies. The objectives of this study were therefore to assess the refill adherence for dispensed oral long-term medications in a random sample of the general adult population in Sweden, and to investigate whether the percentages of self-reported ADRs and STEs differed for medications with adequate refill adherence, oversupply and undersupply. 


\section{METHODS}

\section{Study population}

A sample of 14000 persons aged 18 years and above registered in Sweden on January $1^{\text {st }}$ 2010 was drawn by Statistic Sweden from the Total Population Register, using simple random sampling. ${ }^{7}$ Persons who deceased or migrated between the sampling date and the date of administrating the survey were removed $(n=69)$ and the survey was mailed to 13921 individuals in October 2010.

This study included respondents who were dispensed two or more refills of oral antihypertensive, oral lipid-lowering or oral anti-diabetic medications during the study period. These medications were selected as they are prescribed to a large proportion of the population, are available on prescription only, require long periods of adherent use to achieve the desired outcomes, and their adverse outcomes are well described in studies using clinical data. $^{1,8,9}$ Persons with multidose dispensed medications (ApoDos) were excluded, because information on the prescribed daily dose was missing. Non-interpretable dosage instructions such as "1-2 tablets daily", "2 tablets per day, decrease if side effects" or "1 tablet when needed" (as diuretics for treatment of swallowed legs) were also excluded (Figure 1).

\section{Data sources}

The survey included open and closed questions on experienced ADRs and STEs during the past month, the associated medications and whether the adverse outcomes caused hospitalizations. The questionnaire was developed by the research group based on earlier studies, ${ }^{10,11}$ and was pilot-tested in different populations for face and content validity. Medications reported to be responsible for ADRs or STEs by respondents were coded using Anatomical Therapeutic Chemical classification (ATC). ${ }^{12}$ If a medication class was reported to be responsible for an ADR or STE, individual medications belonging to this class and dispensed to the respondent were considered responsible for the ADR or STE.

Survey responses and registers data were combined using the unique personal identity number. Data on dispensed prescription medications were obtained from the Swedish Prescribed Drug Register (SPDR). ${ }^{13}$ It contains information on the name of the dispensed medications, the ATC code, the amount of medications dispensed, the prescribed daily dose given in a free text field, and the date of dispensing. 
The selected medication classes were defined as the following ATC-codes: i) A10B (oral antidiabetics); ii) C02, C03, C07, C08 and C09 (antihypertensives); iii) and C10 (lipid-lowering drugs). ${ }^{12}$ Information about hospitalizations was retrieved from the National Patient Register, including the date of hospitalization and discharge. Sociodemographic characteristics were retrieved from the LISA database (the longitudinal integration database for health insurance and labour market studies) from Statistics Sweden.

\section{Defining refill adherence}

Refill adherence calculations were performed at the chemical substance level ( $5^{\text {th }}$ ATC level). Dosage instructions given in free text fields were interpreted to determine the number of prescribed daily units. The refill adherence was measured using the continuous measure of medication acquisition (CMA). ${ }^{14,15}$ The CMA was assessed during a 10-month study period, which started ten months before the date of returning the survey and thus included the one month period for the eventual occurrence of an ADR or STE. Because long-term medications are usually dispensed every three months in Sweden, the 10-month period was sufficient to include the minimum of two refills for calculating the CMA.

The CMA was defined as the cumulative number of days' supply divided by the number of days during the 10-month study period. The number of days during the study period was calculated from the index date (first medication dispensing during the study period) until the end of the observation period.

\section{Cumulative number of days' supply \\ $\mathrm{CMA}=\overline{\text { Number of days during the observation period }}$}

To gain the cumulative number of days' supply for each included dispensation, the number of dispensed units was divided by the number of prescribed daily units.

Calculations of refill adherence scenarios are illustrated in Figure 2. If the last refill day exceeded the 10-month observation period, only the number of day's supply included during the observation period were included. If hospitalizations occurred during the study period, medications were assumed to be provided at hospital and the number of hospitalization days was added to the cumulative number of days' supply. 
We applied cut-offs used in previous studies for CMA. Adequate refill adherence to a medication therapy was defined as a CMA between 0.8 and 1.2, oversupply of medication as a CMA $>1.2$ and undersupply of a medication as a CMA $<0.8 .^{16-18} \mathrm{~A}$ person was considered to have an adequate refill adherence if all the medication therapies belonging to the therapeutic class had adequate refill adherence. A person was considered to oversupply or to undersupply if at least one of the refilled medication therapies were oversupplied or undersupplied.

\section{Statistical analysis}

The study population was described by gender, age, hospitalizations during the study period, the mean number of dispensed medications, and which of the studied medications they were dispensed. The CMA was calculated with the median proportion of days with medication therapies and the first and third quartiles. The number and percentage of adequate refill adherence, oversupply and undersupply were reported for the three medication classes and at person-level. The number and percentage of self-reported ADRs and STEs were reported. Pearson's chi-squared tests were used to analyze whether the percentages of self-reported ADRs or STEs differed for medications with adequate refill adherence, oversupply and undersupply. The level for statistical significance was 0.05 .

To assess the stability of the relation between refill rates of medication therapies and the reporting of ADRs and STEs, we performed a sensitivity analysis by considering different categories of refill rates. The percentages of self-reported ADRs and STEs were calculated for stepwise categories of refill rates $(0.2 ; 0.4 ; 0.6 ; 0.8 ; 1 ; 1.2)$, and compared using Pearson's chi-squared tests.

Data management and statistical analysis were performed in STATA version 10.0 (StataCorp, TX) and Excel Microsoft 2007.

\section{Ethical considerations}

The respondents consented to participate to the study by responding to the survey. An introductory letter sent in conjunction with the survey followed the principals of the Declaration of Helsinki. ${ }^{19}$ The sampled persons were informed that their answers would be complemented with data from population registers and that by responding the survey they also 
consented that registers information will be accessed and used. In order to protect the integrity of respondents, Statistics Sweden administered the survey, linked the survey responses to register data, and de-identified the combined dataset. An ethical approval was received from the Regional ethical review board in Gothenburg (registration number: 238-2010).

\section{RESULTS}

Of all respondents $(\mathrm{n}=7099), 30.1 \%$ was dispensed at least one studied medication. Among them, 3.1\% had multi-dose dispensed drugs, $9.8 \%$ had only one refill during the study period, and $1.4 \%$ had non-interpretable dosage instructions, and were therefore excluded (Figure 1).

Finally, 1827 persons were included in the study. The characteristics of the study population are presented in Table 1. The mean age was 68.3 years, $49.1 \%$ was male, $13.7 \%$ was hospitalized during the study period, and the mean number of dispensed medications was seven. Of the included persons, 90.6\% were dispensed antihypertensives, $44.8 \%$ lipid lowering drugs and $11.2 \%$ oral anti-diabetics. Persons dispensed all of the three medication classes represented $5.9 \%$ of the study population.

At medication-level, antihypertensives, lipid lowering drugs and oral anti-diabetics represented respectively $73.7 \%, 20.6 \%$ and $5.7 \%$ of the medication therapies. In total, 4106 medications therapies were included and the median CMA during the 10-month period was 1.02 (Table 2).. With the cutoffs applied to CMA, 65.7\% of the medications had adequate refill adherence, $21.9 \%$ oversupply, and $12.4 \%$ undersupply. At person-level, $56.5 \%$ of persons who refilled antihypertensives, $66.2 \%$ of persons who refilled lipid lowering drugs, and $60 \%$ of persons who refilled oral anti-diabetics had an adequate refill adherence (Table $3)$.

In total, 107 ADRs and 52 STEs were reported representing respectively $2.6 \%$ and $1.3 \%$ of the studied medication therapies (Table 4). The percentages of self-reported ADRs and STEs did not differ between medications with adequate refill adherence, oversupply and undersupply for the three therapeutic classes. These percentages were respectively $2.6 \%$, $2.7 \%$ and $2.1 \%(p>0.5)$ for ADRs, and $1.1 \%, 1.6 \%$ and $1.5 \%(p>0.5)$ for STEs. For lipid lowering drugs and oral anti-diabetics, differences in reporting ADRs or STEs for medications with adequate refill adherence, oversupply and undersupply could not be statistically analyzed 
due to few reported ADRs and STEs. One ADR was reported to cause a hospitalization. In the sensitivity analysis, the percentages of self-reported ADRs and STEs remained stable in multiple stepwise categories of refill rates (Table 5). No STEs and three ADRs were reported for $\mathrm{CMA}<0.6$.

\section{DISCUSSION}

In this study, adequate refill adherence was found in two thirds of the medication therapies. More than half of persons who refilled antihypertensives and about two thirds of individuals who refilled lipid lowering drugs and oral anti-diabetics had adequate refill adherence to all their medications from theses therapeutic classes. Moreover, the percentages of self-reported ADRs and STEs did not differ for medications with adequate refill adherence, oversupply and undersupply.

The CMA rates found in this study were similar to those found in previous studies using the Swedish Prescribed Drug Register. ${ }^{4}{ }^{20}$ High rates of CMA have also been described in other studies measuring refill adherence from prescription databases, but CMAs superior to one have usually been truncated to one. ${ }^{5,6}$ As we purposefully included oversupply as a pattern of refill non adherence, CMAs superior to one were not truncated to one in our study, increasing the median CMA in our study compared to some previous studies. ${ }^{6}$ In previous studies, adherence rates have varied from $35 \%$ to $97 \%$ for antihypertensives, ${ }^{21}$ from $37 \%$ to $80 \%$ for lipid-lowering drugs, ${ }^{22}$ and from $36 \%$ to $93 \%$ for oral anti-diabetics. ${ }^{23}$ Refill Adherence depends on factors such as the study population characteristics and the measurement method used. Although there is no gold standard method to measure the refill adherence using pharmacy claims databases, a previous review has found that CMA is a reliable measure. ${ }^{24}$

To the best of our knowledge; our study is the first investigating the association between refill adherence to long-term medications and self-reporting of ADRs and STEs at the general population level. We did not find evidence of more ADRs when medications were oversupplied, which may be explained by the Swedish reimbursement system. Prescriptions can be refilled when two-thirds of the previous medication period has passed ${ }^{25}$ and the reimbursement increases during a calendar year with the accumulated co-payment which can provide incentives to stockpile and oversupplies, especially in individuals exempted from co- 
payment. However, we did not control for co-payment. A previous Swedish study has found that oversupply was a leading cause to return unused dispensed medications, ${ }^{26}$ and that about half of returned packages were unused. ${ }^{27}$ Even if oversupply may not lead to higher occurrence of ADRs, it remains a source of extra high costs and wastage for the health care. ${ }^{16}$

Furthermore, we did not find STEs more common when medications were undersupplied, indicating that other factors than refill adherence estimated from prescription databases influence STEs. Although the evidence of the association between underuse of long-term medications and STEs is established from controlled clinical trials and observational studies, ${ }^{28,29}$ the complexity of this association in the clinical practice has been highlighted in systematic reviews and meta-analysis. ${ }^{29-31}$ For the management of cardiovascular diseases, diabetes and hyperlipidemia, the adherence to recognized healthy behaviors has a great impact on the improvement of medication and health outcomes. Even in previous controlled clinical trials, a better adherence to placebo has been associated with better medication related outcomes, which shows that the medication adherence is a marker of overall individual's healthy behaviors. ${ }^{29}$

Nonetheless, the absence of association between refill non adherence and adverse outcomes of medications may have been contributed by underreporting of ADRs and STEs, in particular if the underreporting was disproportional between adherent and non adherent persons. The percentage of self-reported STEs might have been underestimated for the three long-term medications since the associated diseases can remain asymptomatic and individuals could have failed to identify disease symptoms associated with STEs. ${ }^{8}$ Compared to previous studies, $^{32,}{ }^{33}$ our percentage of self-reported ADRs was also low, as individuals might be unsure if the medication in fact caused the symptom. It has been found that patients may experience an ADR for a long time before informing health practitioners. ${ }^{33}$ The low percentages of self-reported ADRs and STEs could reflect a lack of information received by individuals for symptoms of ADRs and STEs. Thus, there may be a need for better communication and adaptation of information given by the caregivers especially for individuals with chronic conditions. Although self-reports are a useful source of information on medication-related morbidities that reflect patients' perception on their medications and 
diseases, ${ }^{34}$ It would be interesting in a future study to compare the association between adherence and ADRs or STEs detected from medical records and self-reports.

The absence of association between refill non adherence and self-reported adverse outcomes of medications in this study suggests that a better understanding of patients' refill adherence behaviors and their perceived adverse outcomes from their long-term medications is needed and should be considered in improving the medication management. Numerous studies have concluded that patients' perspectives should be considered in order to improve medication management, because individuals' views, understanding and perceptions of their medications and their medication-related adverse outcomes affect their refill and intake behavior. ${ }^{35}$ In clinical trials, participants are selected, receive high attention and are informed about their health conditions and the adverse outcomes of their medications, and this may not reflect clinical practice. ${ }^{3}$ An analysis of factors that could have influenced both the self-reporting of medication-related adverse outcomes and the refill adherence would be interesting to investigate. Due to the low number of reported ADRs and STEs, we were unable to analyze the association between the reporting of medication related adverse outcomes and refill adherence using multivariable models with adjustment to the respondents' characteristics.

When interpreting this study some limitations should be considered. A response bias might have been present. Individuals with severe medical conditions might not have answered the survey. However, these individuals would require multidose dispensed medications; therefore their refill adherence would not be possible to calculate. Another limitation is that our study population mainly represents elderly, but this was expected due to the studied medications.

Similar to other refill adherence studies, medication consumption was assumed, and the timing of the doses of medication was unknown. Nevertheless, we used dispensing and not prescription data and the act of refilling a medication reflects a patient's active decision to continue with medication. ${ }^{20}$ One strength of our study is that adherence was not self-reported as non-adherent individuals tend to describe their medication's intake behaviors inaccurately, which would blur any differences of adherence. ${ }^{36}$

Although we aimed to investigate whether the percentages of self-reported ADRs and STEs differed for medications with adequate refill adherence, oversupply and undersupply, respondents might have reported ADRs or STEs that occurred over the year and that could be 
the reason of their refill non adherence. However, because of the low number of reported ADRs and STEs, this was unlikely to change the refill adherence found in our study. It was not possible to verify whether individuals were prescribed the appropriate medications for their health conditions.

Finally, the extent to which our findings are generalizable to all individuals taking the studied long-term medications is unknown. However, our study was comprehensive as the sample was drawn from the whole Swedish adult population.

\section{CONCLUSIONS}

Adequate refill adherence was found in two thirds of the medication therapies. ADRs and STEs were unexpectedly equally commonly reported for medications with adequate refill adherence, oversupply and undersupply, which was possibly contributed by the low percentages of ADRs and STEs. These results suggest that a better understanding of patients' refill behaviors and their perceived adverse outcomes from their long-term medications is needed and should be considered in improving medication management. The impact of individual and healthcare factors that may influence the association between refill adherence and self-reported medication-related adverse outcomes should be investigated in future studies.

\section{Acknowledgements}

The study was conducted as part of the project Drug-Related Morbidity in Sweden (DRUMS). The authors thank the other persons from the research group; in particular Hanna Gyllensten for her comments on the research protocol and Tatiana Sandström for her contribution to data management. 
FIGURES AND TABLES

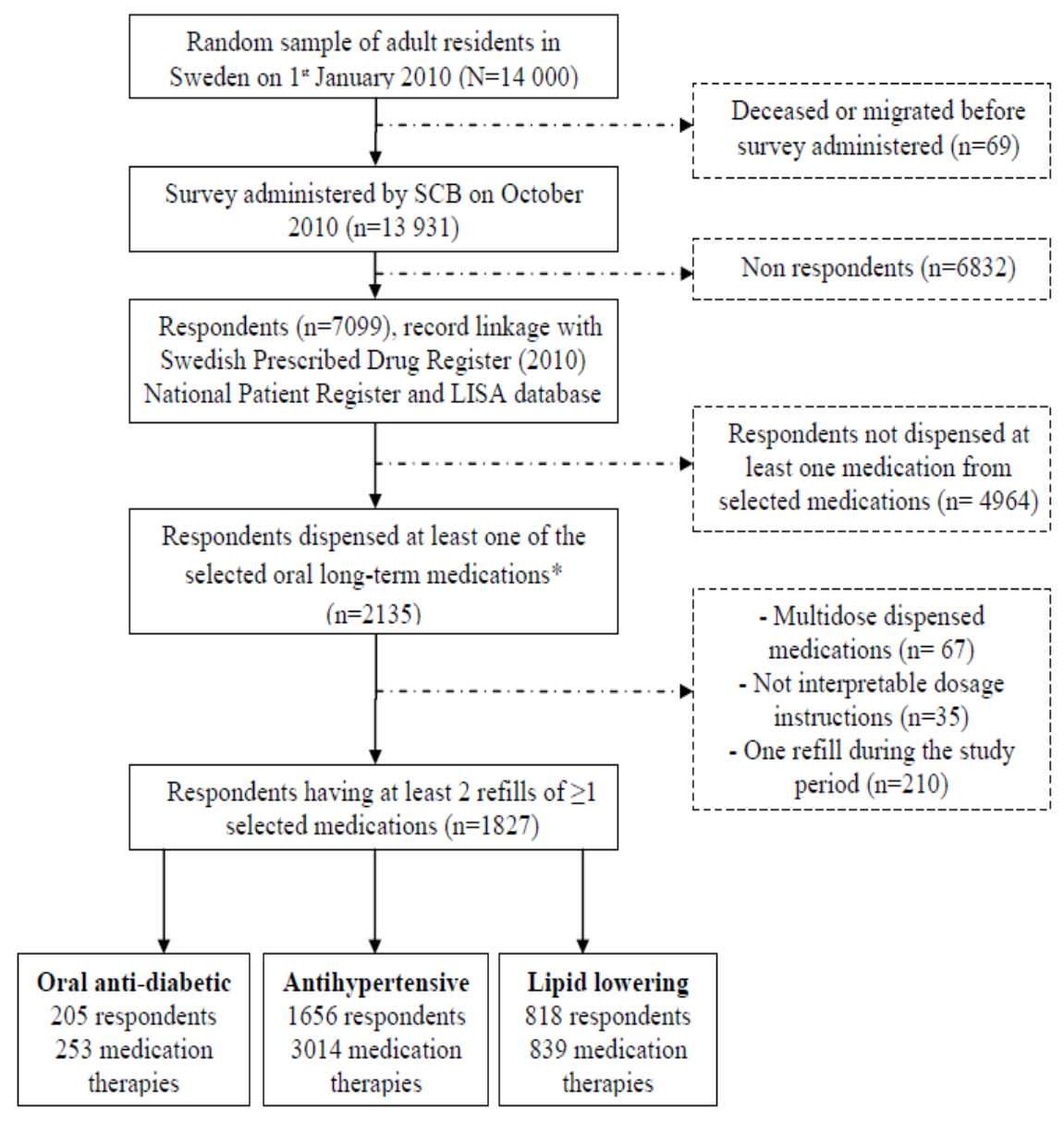

SCB: Statistics Sweden

LISA: The longitudinal integration database for health insurance and labour market studies

*ATC codes: A10B (oral anti-diabetics)/ $\mathrm{C} 02, \mathrm{C} 03, \mathrm{C} 07, \mathrm{C} 08$ and $\mathrm{C} 9$ (antihypertensives)/

C10 (lipid-lowering drugs)

\section{Figure 1: Study flow-diagram}




\section{The last refill does not exceed the 10 -month study period}

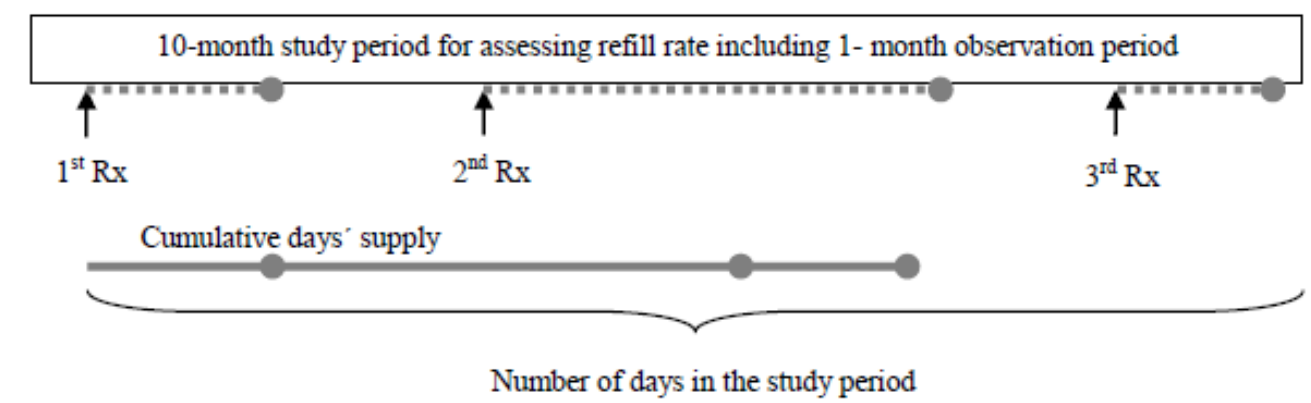

The last refill exceeds the 10 -month study period

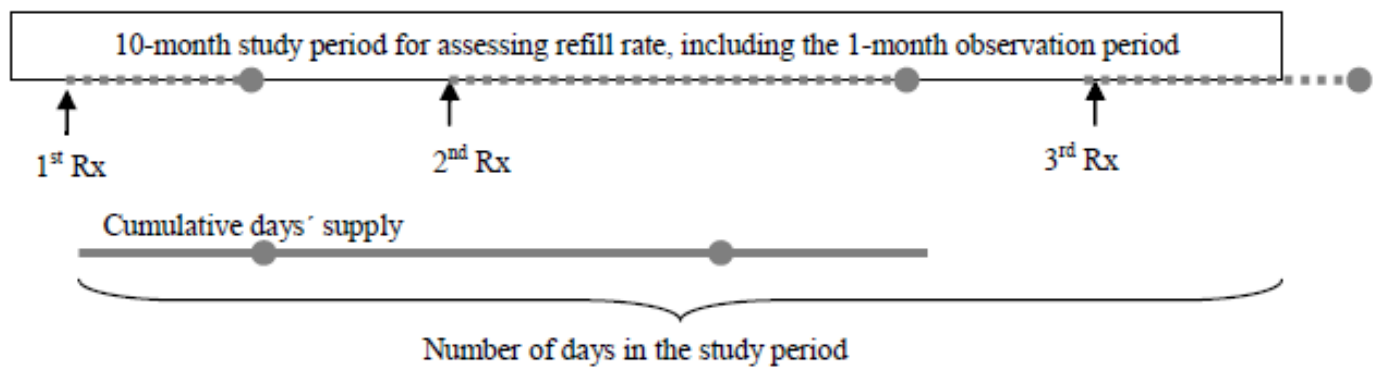

Hospitalization occurring during the 10 -month study period

10 -month study period for assessing refill rate, including the 1 -month observation period

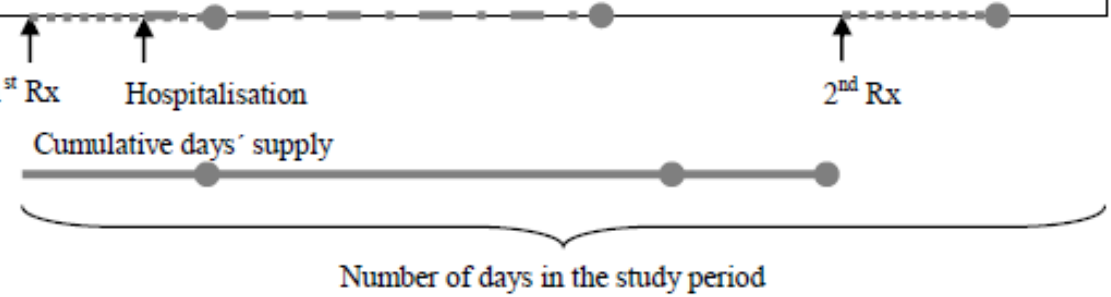

Rx: Prescription refill

The observation period ends the date of returning the survey

Figure 2: Scenarios for the calculation of refill adherence 
Table 1. Study population characteristics $(\mathrm{N}=1827)$

\begin{tabular}{ll}
\hline Characteristics & \\
\hline Gender male, $n(\%)$ & $898(49.1)$ \\
Age, mean (SD) & $68.3(11.4)$ \\
Age years, $n(\%)$ & $20(1.1)$ \\
$18-39$ & $89(4.9)$ \\
$40-49$ & $261(14.3)$ \\
$50-59$ & $630(34.5)$ \\
$60-69$ & $505(27.6)$ \\
$70-79$ & $321(17.6)$ \\
$80-96$ & $251(13.7)$ \\
Hospitalization during the study period, $n(\%)$ & $4(2-10)$ \\
Median length of hospitalization (Q1-Q3) & $7(4)$ \\
Mean number of dispensed medications, $n$ (SD) & $929(50.8)$ \\
Dispensed AHT only, $n(\%)$ & $561(30.7)$ \\
Dispensed AHT and LL, $n(\%)$ & $133(7.3)$ \\
Dispensed LL only, $n(\%)$ & $109(5.9)$ \\
Dispensed AHT, LL and OAD, $n(\%)$ & $56(3.1)$ \\
Dispensed AHT and OAD, $n(\%)$ & $26(1.4)$ \\
Dispensed OAD only, $n(\%)$ & $14(0.8)$ \\
Dispensed LL and OAD, $n(\%)$ & \\
\hline SD: Standard deviation; Q1-Q3: First and third quartiles; AHT: Antihypertensive medications; \\
LL: Lipid lowering; OAD: Oral anti-diabetics
\end{tabular}


Table 2. Refill adherence of oral long-term medication therapies $(\mathrm{N}=4106)$, per medication class

\begin{tabular}{|c|c|c|c|c|c|}
\hline \multirow[b]{2}{*}{ Medical therapy } & \multirow{2}{*}{$\begin{array}{c}\text { CMA } \\
\text { Proportion of } \\
\text { days with } \\
\text { medications } \\
\text { median }(\mathbf{Q} 1-\mathrm{Q} 3)\end{array}$} & \multicolumn{4}{|c|}{ Refill adherence } \\
\hline & & $\begin{array}{c}\text { Adequate } \\
\text { refill } \\
\text { adherence*n } \\
(\%)\end{array}$ & $\begin{array}{c}\text { Oversupply } \dagger \\
n(\%)\end{array}$ & $\begin{array}{c}\text { Undersupply } \\
+\leftarrow \\
n(\%)\end{array}$ & Total \\
\hline Total & $1.02(0.92-1.16)$ & $2699(65.7)$ & $901(21.9)$ & $506(12.4)$ & 4106 \\
\hline Antihypertensives & $1.02(0.93-1.17)$ & $1974(65.5)$ & $688(22.8)$ & $352(11.7)$ & 3014 \\
\hline Lipid lowering drugs & $1.00(0.88-1.13)$ & $559(66.6)$ & $154(18.4)$ & $126(15.1)$ & 839 \\
\hline Oral anti-diabetics & $1.00(0.95-1.18)$ & $166(65.6)$ & $59(23.3)$ & $28(11.1)$ & 253 \\
\hline
\end{tabular}


Table 3. Percentages of persons with adequate refill adherence, oversupply and undersupply $(\mathrm{N}=1827)$

\begin{tabular}{|c|c|c|c|c|c|}
\hline Persons dispensed & $\begin{array}{c}\text { Adequate } \\
\text { refill } \\
\text { adherence* } \\
n(\%)\end{array}$ & $\begin{array}{c}\text { Oversupply } \dagger \\
n(\%)\end{array}$ & $\begin{array}{c}\text { Undersupply } \\
n(\%)\end{array}$ & $\begin{array}{c}\text { Both } \\
\text { undersupply } \\
\text { and } \\
\text { oversupply } \$ \\
n(\%)\end{array}$ & Total \\
\hline Antihypertensives & $937(56.6)$ & $435(26.3)$ & $225(13.6)$ & $59(3.5)$ & 1656 \\
\hline Lipid lowering drugs & $542(66.2)$ & $152(18.6)$ & $122(14.9)$ & $2(0.3)$ & 818 \\
\hline Oral anti-diabetics & $123(60.0)$ & $54(26.3)$ & $25(12.2)$ & $3(1.5)$ & 205 \\
\hline
\end{tabular}

*Adequate refill adherence: Cumulative medication Acquisition (CMA) of all medications from the therapeutic class $\geq 0.8$ and $\leq 1.2$

$\dagger$ Oversupply: At least one medication from the therapeutic class with CMA $>1.2$

tUndersupply: At least one medication from the therapeutic class with CMA $<0.8$

§oth undersupply and oversupply: At least one medication from the therapeutic class with CMA $<0.8$ and another medication from the same therapeutic class with CMA $>1.2$ 
Table 4. Percentages of self-reported ADRs and STEs for medication therapies with adequate refill adherence, oversupply and undersupply $(\mathrm{N}=4106)$

\begin{tabular}{|c|c|c|c|c|}
\hline & \multicolumn{2}{|c|}{ ADRs } & \multicolumn{2}{|c|}{ STES } \\
\hline & $n(\%)$ & $\begin{array}{c}\text { Pearson's } \chi^{2} \\
\text { test }\end{array}$ & $n(\%)$ & $\begin{array}{c}\text { Pearson's } \chi^{2} \\
\text { test }\end{array}$ \\
\hline Total & $107(2.6)$ & & $52(1.3)$ & \\
\hline Adequate refill adherence* & $71(2.6)$ & $p>0.5 \S$ & $30(1.1)$ & $p>0.5 \S$ \\
\hline Oversupply† & $25(2.7)$ & & $15(1.6)$ & \\
\hline Undersupplyt & $11(2.1)$ & & $7(1.5)$ & \\
\hline \multicolumn{5}{|l|}{ Antihypertensives } \\
\hline Adequate refill adherence & $57(2.9)$ & $p>0.5 \S$ & $25(1.3)$ & $p>0.5 \S$ \\
\hline Oversupply & $22(3.1)$ & & $11(1.6)$ & \\
\hline Undersupply & $8(2.3)$ & & $5(1.7)$ & \\
\hline \multicolumn{5}{|l|}{ Lipid lowering drugs } \\
\hline Adequate refill adherence & $7(1.2)$ & NA & $0(0.0)$ & NA \\
\hline Oversupply & $2(1.3)$ & & $1(0.6)$ & \\
\hline Undersupply & $2(1.6)$ & & $1(0.7)$ & \\
\hline \multicolumn{5}{|l|}{ Oral anti-diabetics } \\
\hline Adequate refill adherence & $7(4.2)$ & NA & $5(3.0)$ & NA \\
\hline Oversupply & $1(1.7)$ & & $3(3.5)$ & \\
\hline Undersupply & $1(3.6)$ & & $1(5.0)$ & \\
\hline $\begin{array}{l}\text { ADR: Adverse drug reactic } \\
* \text { Adequate refill adherence: } \\
\text { †Oversupply: CMA }>1.2 \\
\text { †Undersupply: CMA }<0.8 \\
\text { §Non significant difference }\end{array}$ & $\begin{array}{l}\text { STE: Sub-t } \\
\text { umulative } n\end{array}$ & $\begin{array}{l}\text { erapeutic effec } \\
\text { edication Acqu }\end{array}$ & $\begin{array}{l}\chi^{2}: \text { Chi-sq } \\
\text { ition }(\mathrm{CM}\end{array}$ & $\begin{array}{l}\text { ared; NA: Not } \\
\geq 0.8 \text { and } \leq 1\end{array}$ \\
\hline
\end{tabular}


Table 5. Percentages of self-reported ADRs and STEs for medication therapies in stepwise categories of refill rates $(\mathrm{N}=4106)$

\begin{tabular}{cccccc}
\hline & & \multicolumn{2}{c}{ ADRs } & \multicolumn{2}{c}{ STEs } \\
\cline { 3 - 6 } CMA & $\mathbf{N}$ & $\boldsymbol{n}(\boldsymbol{\%})$ & $\begin{array}{c}\text { Pearson's } \boldsymbol{\chi}^{2} \\
\text { test }\end{array}$ & $\boldsymbol{n}(\boldsymbol{\%})$ & $\begin{array}{c}\text { Pearson's } \boldsymbol{\chi}^{2} \\
\text { test }\end{array}$ \\
\hline$<0.8^{*}$ & 506 & $10(2.0)$ & $p>0.05 \dagger$ & $6(1.2)$ & $p>0.05 \dagger$ \\
$0.8-1$ & 1241 & $34(2.7)$ & & $9(0.7)$ & \\
$1-1.2$ & 1458 & $38(2.6)$ & & $22(1.5)$ & \\
$>1.2$ & 901 & $25(2.8)$ & & $15(1.7)$ & \\
Total & $\mathbf{4 1 0 6}$ & $\mathbf{1 0 7}(\mathbf{2 . 6})$ & & $\mathbf{5 2}(\mathbf{1 . 3})$ & \\
\hline
\end{tabular}

CMA: Continuous measure of medication acquisition; ADR: Adverse drug reaction STE: Sub-therapeutic effect; $\chi^{2}$ : Chi-squared

*No STE and three ADRs were reported for CMA $<0.6$

$\dagger$ Non significant difference 


\section{REFERENCES}

1.World Health Organization. Adherence to long-term therapies: Evidence for action. Geneva. 2003.

2.Cramer JA, Roy A, Burrell A, et al. Medication compliance and persistence: Terminology and definitions. Value Health. 2008; 11: 44-47.

3.McDonald HP, Garg AX, Haynes RB. Interventions to enhance patient adherence to medication prescriptions: Scientific review. JAMA. 2002; 288: 2868-2879.

4.Lesen E, Sandstrom TZ, Carlsten A, et al. A comparison of two methods for estimating refill adherence to statins in Sweden: The RARE project. Pharmacoepidemiol Drug Saf. 2011; 20: 1073-1079.

5. Yeaw J, Benner JS, Walt JG, et al. Comparing adherence and persistence across 6 chronic medication classes. J Manag Care Pharm. 2009; 15: 728-740.

6.Briesacher BA, Andrade SE, Fouayzi H, et al. Comparison of drug adherence rates among patients with seven different medical conditions. Pharmacotherapy. 2008; 28: 437-443.

7.Statistics Sweden. Sampling - from theory to practice [in Swedish]. 2008.

8.McDonald M, Hertz RP, Unger AN, et al. Prevalence, awareness, and management of hypertension, dyslipidemia, and diabetes among united states adults aged 65 and older. $J$ Gerontol A Biol Sci Med Sci. 2009; 64: 256-263.

9.Cramer JA, Benedict A, Muszbek N, et al. The significance of compliance and persistence in the treatment of diabetes, hypertension and dyslipidaemia: A review. Int J Clin Pract. 2008; 62: 76-87.

10.Isacson D, Johansson L, Bingefors K. Nationwide survey of subjectively reported adverse drug reactions in sweden. Ann Pharmacother. 2008; 42: 347-353.

11.Gandhi TK, Weingart SN, Borus J, et al. Adverse drug events in ambulatory care. $N$ Engl $J$ Med. 2003; 348: 1556-1564.

12.World Health Organization. Guidelines for ATC Classification and DDD Assignment WHO. Collaborating Centre for Drug Statistics Methodology; 2012.

13. Wettermark B, Hammar N, Fored CM, et al. The new Swedish prescribed drug registeropportunities for pharmacoepidemiological research and experience from the first six months. Pharmacoepidemiol Drug Saf. 2007; 16: 726-735.

14.Steiner JF, Prochazka AV. The assessment of refill compliance using pharmacy records: Methods, validity, and applications. J Clin Epidemiol. 1997; 50: 105-116. 
15.Caetano PA, Lam JM, Morgan SG. Toward a standard definition and measurement of persistence with drug therapy: Examples from research on statin and antihypertensive utilization. Clin Ther. 2006; 28: 1411-24.

16.Krigsman K, Melander A, Carlsten A, et al. Refill non-adherence to repeat prescriptions leads to treatment gaps or to high extra costs. Pharm World Sci. 2007; 29: 19-24.

17.Dilokthornsakul P, Chaiyakunapruk N, Nimpitakpong P, et al. The effects of medication supply on hospitalizations and health-care costs in patients with chronic heart failure. Value Health. 2012; 15: S9-14.

18. Stroupe KT, Teal EY, Tu W, et al. Association of refill adherence and health care use among adults with hypertension in an urban health care system. Pharmacotherapy. 2006 ;26: 779-789.

19.World Medical Association. Declaration of Helsinki Ethical Principles for Medical Research Involving Human Subjects. Available at: http://www.wma.net/fr/30publications/10policies/b3/index.html.pdf. Accessed 0/06, 2013.

20.Haupt D, Weitoft GR, Nilsson JL. Refill adherence to oral antihyperglycaemic drugs in sweden. Acta Diabetol. 2009; 46: 203-208.

21.Ostchega Y, Yoon SS, Hughes J, et al. Hypertension awareness, treatment, and control-continued disparities in adults: United states, 2005-2006. NCHS Data Brief. 2008; 3:1-8.

22.Schedlbauer A, Davies P, Fahey T. Interventions to improve adherence to lipid lowering medication. Cochrane Database Syst Rev. 2010:3.

23.Cramer JA. A systematic review of adherence with medications for diabetes. Diabetes Care. 2004; 27: 1218-1224.

24.Hess LM, Raebel MA, Conner DA, et al. Measurement of adherence in pharmacy administrative databases: A proposal for standard definitions and preferred measures. Ann Pharmacother. 2006; 40: 1280-1288.

25.Act 2003:460. Concerning the ethical review of research involving humans [In Swedish].

26.Ekedahl AB. Reasons why medicines are returned to Swedish pharmacies unused. Pharm World Sci. 2006; 28: 352-358.

27.Ekedahl A, Wergeman L, Rydberg T. Unused drugs in Sweden measured by returns to pharmacies. J Soc Adm Pharm. 2003; 20: 26--31.

28.Ho PM, Rumsfeld JS, Masoudi FA, et al. Effect of medication nonadherence on hospitalization and mortality among patients with diabetes mellitus. Arch Intern Med. 2006; 166: $1836-1841$.

29.Simpson SH, Eurich DT, Majumdar SR, et al. A meta-analysis of the association between adherence to drug therapy and mortality. BMJ. 2006; 333: 15. 
30.Haynes RB, McDonald HP, Garg AX. Helping patients follow prescribed treatment: Clinical applications. JAMA. 2002; 288: 2880-2883.

31.DiMatteo MR, Giordani PJ, Lepper HS, et al. Patient adherence and medical treatment outcomes: A meta-analysis. Med Care. 2002; 40: 794-811.

32.Oladimeji O, Farris KB, Urmie JG, et al. Risk factors for self-reported adverse drug events among medicare enrollees. Ann Pharmacother. 2008; 42: 53-61.

33.Weingart SN, Gandhi TK, Seger AC, et al. Patient-reported medication symptoms in primary care. Arch Intern Med. 2005; 165: 234-240.

34.Avery AJ, Anderson C, Bond CM, et al. Evaluation of patient reporting of adverse drug reactions to the UK 'yellow card scheme': Literature review, descriptive and qualitative analyses, and questionnaire surveys. Health Technol Assess. 2011; 15: m1-234.

35.Horne R, Weinman J. Patients' beliefs about prescribed medicines and their role in adherence to treatment in chronic physical illness. J Psychosom Res. 1999; 47: 555-567.

36.Stephenson $\mathrm{BJ}$, Rowe $\mathrm{BH}$, Haynes $\mathrm{RB}$, et al. The rational clinical examination. Is this patient taking the treatment as prescribed? JAMA. 1993; 269: 2779-2781. 